\title{
Multicentric Type 3 Gastric Neuroendocrine Tumors
}

\author{
Sang Hoon Lee ${ }^{1}$, Dochang Moon ${ }^{1}$, Hee Seung Lee ${ }^{1}$, Choong-kun Lee ${ }^{1}$, Yong Duk Jeon ${ }^{1}$, Ji Hye Park ${ }^{2}$, Hyunki Kim ${ }^{3}$ \\ and Sang Kil Lee ${ }^{1,3}$ \\ ${ }^{1}$ Department of Internal Medicine, Yonsei University College of Medicine, Seoul, ${ }^{2}$ Department of Pathology, Severance Hospital, Yonsei \\ University College of Medicine, Seoul, ${ }^{3}$ Institute of Gastroenterology, Yonsei University College of Medicine, Seoul, Korea
}

A 50-year-old woman with incidentally detected multiple gastric polyps and biopsy-proven neuroendocrine tumor (NET) was referred to our hospital. More than 10 polypoid lesions (less than $15 \mathrm{~mm}$ ) with normal gastric mucosa were detected from the gastric body to the fundus. The serum level of gastrin was within the normal limits. There was no evidence of atrophic changes on endoscopy and serologic marker as pepsinogen I/II ratio. Computed tomography of the abdomen and pelvis revealed no evidence of metastatic lesions. She refused surgery, and we performed endoscopic polypectomy for almost all the gastric polyps that were greater than $5 \mathrm{~mm}$. Although the histological examination revealed that all the removed polys were diagnosed as NET G1, three of them extended to the lateral or vertical resection margins, while two exhibited lymphovascular invasion. A follow-up upper endoscopy that was performed 6 months after the diagnosis showed multiple remnant gastric polyps that were suggestive of remnant gastric NET. Clin Endosc 2015;48:431-435

Key Words: Neuroendocrine tumors; Stomach; Multicentric; Type 3

\section{INTRODUCTION}

Gastric neuroendocrine tumors (NETs), previously referred to as gastric carcinoids, are rare neoplasms that account for $0.6 \%$ to $2 \%$ of all identified gastric polyps and $8.7 \%$ of all gastrointestinal NETs. ${ }^{1,2}$ The incidence of gastric NETs has increased over the last 50 years, partly due to the improvement of endoscopic surveillance and heightened awareness. ${ }^{1,3}$

Gastric NETs are usually classified into three types based on the background gastric pathology. Type 1 arises in the presence of achlorhydric atrophic body gastritis (ABG), type 2 is associated with the Zollinger-Ellison syndrome (ZES) and multiple endocrine neoplasia type 1 (MEN-1), while type 3 includes the "sporadic" NET in the absence of a specific back-

Received: September 19, 2014 Revised: October 24, 2014

Accepted: October 24, 2014

Correspondence: Sang Kil Lee

Department of Internal Medicine, Yonsei University College of Medicine, 50 Yonsei-ro, Seodaemun-gu, Seoul 03722, Korea

Tel: +82-2-2228-1996, Fax: +82-2-393-6884, E-mail: SKLEE@yuhs.ac

(c) This is an Open Access article distributed under the terms of the Creative Commons Attribution Non-Commercial License (http://creativecommons.org/ licenses/by-nc/3.0) which permits unrestricted non-commercial use, distribution, and reproduction in any medium, provided the original work is properly cited. ground of pathologic changes. ${ }^{4}$ This classification plays an important clinical role when approaching a gastric NET, not only due to the possible coexistence of a predisposing condition such as ABG and ZES, but also due to the implications in tumor behavior and the prognosis of the patient.

Type 3 gastric NET represents $14 \%$ to $25 \%$ of gastric NETs and have poor prognosis with the highest rate of distant metastasis. ${ }^{5,6}$ They mostly are large $(>2 \mathrm{~cm})$ solitary lesions arising in the normal surrounding mucosa. ${ }^{7}$ In this case, we describe a patient with multicentric (more than 10) small gastric NETs identified as type 3 .

\section{CASE REPORT}

A 50-year-old woman underwent an esophagogastroduodenoscopy (EGD) for a health check-up. More than 10 polypoid lesions of the stomach were discovered, and the biopsy specimens from the gastric polyps demonstrated grade 1 (G1) NET, according to World health Organization classification. ${ }^{8}$ She was referred to our hospital for proper management of the gastric NETs.

She had no carcinoid symptoms such as flushes or diarrhea, 

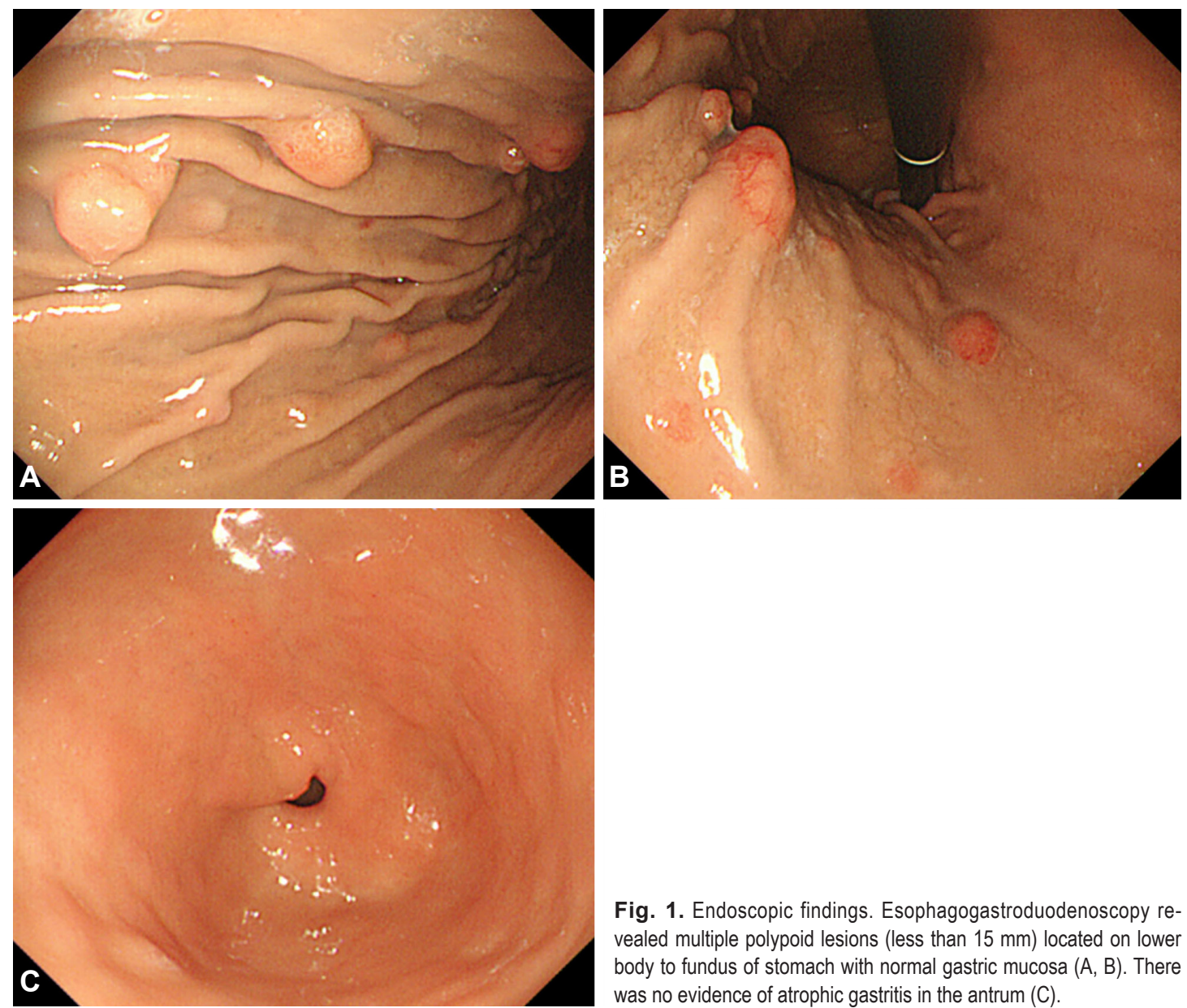

Fig. 1. Endoscopic findings. Esophagogastroduodenoscopy revealed multiple polypoid lesions (less than $15 \mathrm{~mm}$ ) located on lower body to fundus of stomach with normal gastric mucosa (A, B). There was no evidence of atrophic gastritis in the antrum (C).

had no significant medical history, and did not previously receive any proton pump inhibitors or $\mathrm{H}_{2}$ receptor antagonists. Interestingly, her sister also had gastric NETs. The results of the peripheral blood and routine chemistry tests were within the normal limits. The serum level of fasting gastrin was 85.2 $\mathrm{pg} / \mathrm{mL}$ (normal rage, 0 to 90 ). The serum level of pepsinogen I (PG I) was $70.7 \mathrm{ng} / \mathrm{mL}$ and that of pepsinogen II (PG II) was $10.9 \mathrm{ng} / \mathrm{mL}$ with the PG I/II ratio being 6.48 (positive range, $\mathrm{PG}$ I $\leq 70 \mathrm{ng} / \mathrm{mL}$ and PG I/II $\leq 3.0$ ratio). Helicobacter pylori were not detected with rapid urease test and anti- $H$. pylori immunoglobulin $\mathrm{G}$ antibody level was $9.1 \mathrm{AU} / \mathrm{mL}$ with equivocal range (negative range, $<8.0 \mathrm{AU} / \mathrm{mL}$ ). On EGD (A5 CE0 mode, GIF-Q260 scope; Olympus Optical, Tokyo, Japan), multiple polypoid lesions were detected mainly around the greater curvature of the gastric body to the fundus. Some polyps accompanied the erythematous mucosal change, and the maximum diameter of polyps was less than $15 \mathrm{~mm}$ (Fig. 1A, B). Focal granular mucosal change was detected in the gastric body, but there was no evidence of atrophic gastritis in the antrum (Fig. 1C). A computed tomography scan of the abdomen and pelvis revealed multiple enhancing polypoid lesions in the stomach without any evidence of metastatic lesions.

She refused surgery, and we decided to perform endoscopic polypectomy. Polypectomy was performed without complications and almost all the gastric polyps that were greater than $5 \mathrm{~mm}$ in size were removed. A histological examination revealed that all the removed polys were NET GI, which was composed of uniform cells with round or ovoid nuclei and scanty eosinophilic cytoplasm, proliferating in a trabecular or glandular pattern (Fig. 2). The tumor cells invaded the submucosal layer, diffusely staining for chromogranin A. The mitotic count was absent and the Ki-67 index was less than $1 \%$. Most significantly, three of the polyps extended to the lateral or vertical resection margins and two exhibited lymphovascular invasion. Fundic gland atrophy was not detected from random biopsies on the greater curvature of the upper body, mid-body, and antrum. We diagnosed this patient with multicentric type 3 gastric NETs. After the procedure, she still refused surgery despite the high risk of metastasis and tumor-related death. Follow-up EGD at 6 months after diagnosis showed multiple remnant gastric polyps suggestive of gastric NETs (Fig. 3). 


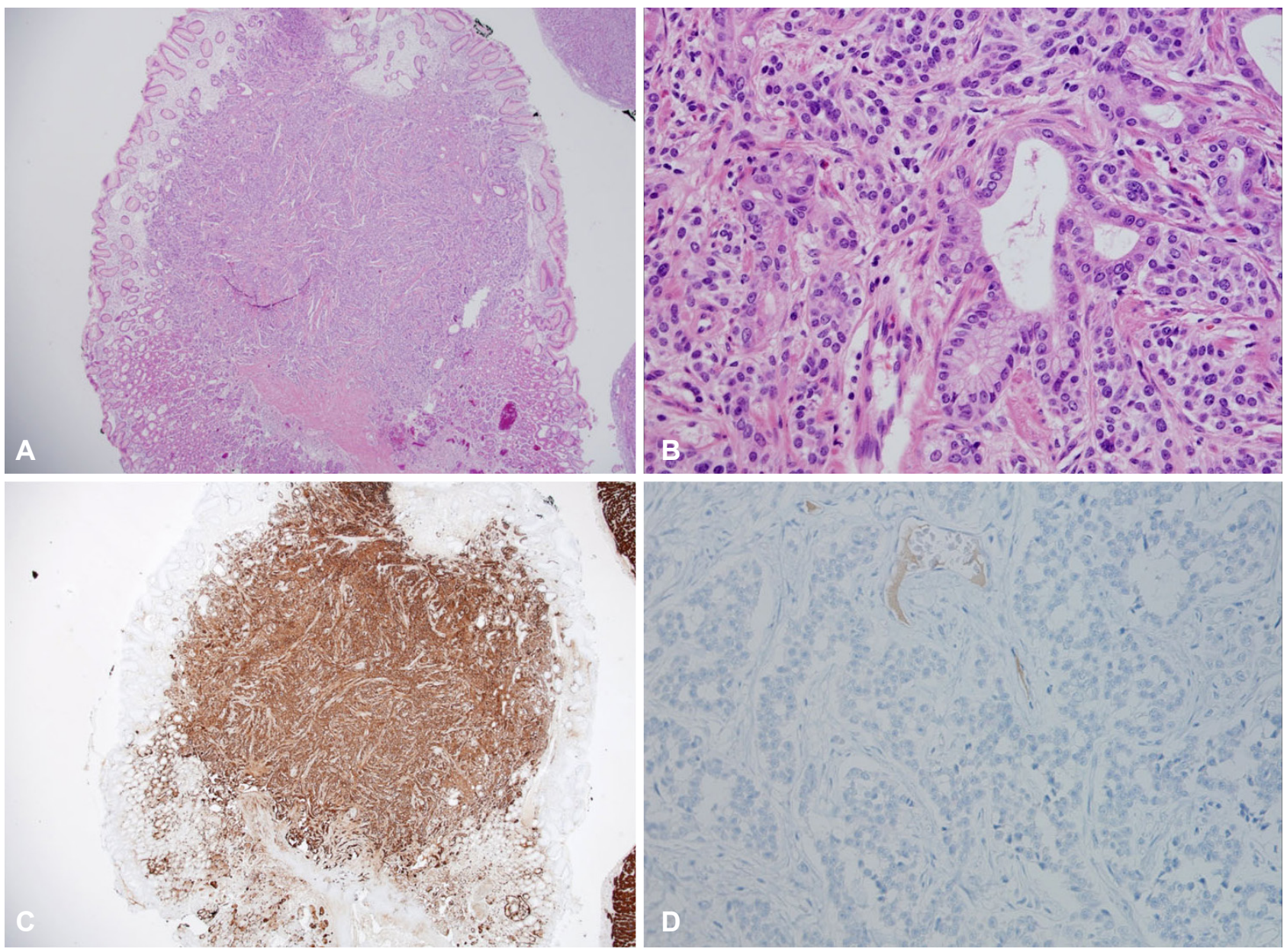

Fig. 2. Histological examination of the gastric neuroendocrine tumor. Hematoxylin and eosin staining (H\&E stain) showed that tumor cells invaded into the submucosal layer $(A, \times 40)$. The tumor was composed of uniform cells with round or ovoid nuclei and scanty eosinopohlic cytoplasm, proliferating in a trabecular or glandular pattern, which were absent of mitotic count $(B, \times 100)$. Immunohistochemical stating for chromogranin $A$ was diffusely positive $(C, \times 40)$. The Ki-67 labeling index was less than $1 \%(D, \times 100)$.

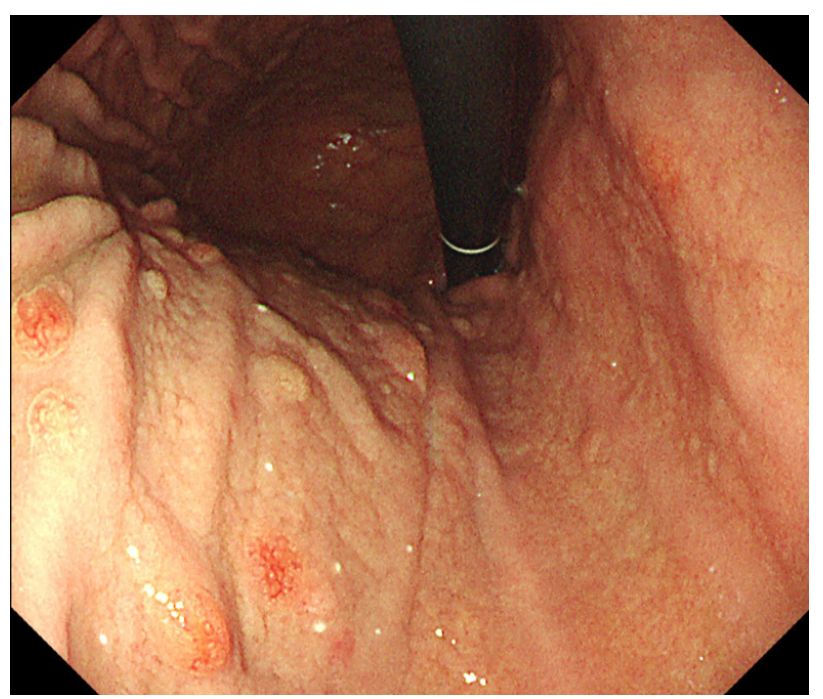

Fig. 3. Follow-up endoscopic findings. Esophagogastroduodenoscopy after 6 months from diagnosis still showed multiple remnant gastric polyps.

\section{DISCUSSION}

Gastric NETs were first categorized into three types in 1993 by Rindi et al. ${ }^{4}$ Type 1 and 2 are related to the presence of hypergastrinemia causing hyperplasia of the precursor enterochromaffin-like (ECL) cells, whereas type 3 occurs sporadically and independently of gastrin. This classification is based on the clinical differences of epidemiological, pathophysiological, endoscopic, and histological features between each type that affects prognosis, management, and follow-up. ${ }^{9}$ Type 1 and 2 gastric NET have indolent behaviors, but type 3 gastric NET may be life-threatening with a high risk of metastasis and tumor-related death.?

In type 1 and 2 gastric NET, hypergastrinemia plays a crucial role in the development of tumors. ${ }^{10}$ The ECL cells, located in the corpus-fundus mucosa of the stomach, represent the major proliferative target of gastrin. Proliferation of the ECL 
cells results in tumorigenesis of NET. Gastric NET arising from these conditions grows usually multicentric lesions. On the other hand, types 3 gastric NETs are "gastrin-independent" tumors that are rarely multiple. ${ }^{4}$

Endoscopically, type 1 gastric NET tumors are often found in the fundus of stomach and are mostly polypoid (78\%), of small shape (size 5 to $8 \mathrm{~mm}$ ), and are multicentric (68\%; mean number, 3). ${ }^{11,12}$ Type 2 gastric NETs are also usually identified as small, often multiple, polypoid tumors $(<1 \mathrm{~cm}$ in size $)$ in fundus. ${ }^{13}$ On the contrary, a type 3 gastric NET is typically a large (>1 cm, 66\%), solitary (96\%) lesion, that grows from the gastric body/fundus, and occurs in the antrum, within the context of a normal gastric mucosa. ${ }^{14,15}$ In this case, the endoscopic findings of multicentric ( $>10$ lesions), small $(<2 \mathrm{~cm})$ polypoid lesions in the gastric fundus resembled closely to the type 1 and 2 gastric NETs. However, the diagnosis of type 3 gastric NET was clinically based on normal gastrin levels and the absence of evidence of gastric atrophy or significant peptic ulcers that are commonly seen with type 1 or 2 gastric NETs, respectively.

A few cases of multiple gastric NETs without hypergastrinemia have been reported. Some cases of multiple gastric NETs in MEN 1 patients without hypergastrinemia have been previously described. ${ }^{16-18}$ These cases suggest that the development of gastric NET in MEN 1 patients arises not only from hypergastrinemia in ZES, but also from the genetic mutations of the MEN 1 gene, one of the tumor suppressor genes. Moriyama et al. ${ }^{19}$ first reported multicentric gastric NETs that have been classified as type 3 . In this case, the patient concomitantly had a pituitary tumor, an adenomatous goiter, and bilateral Warthin's tumors. Although a possible predisposition to MEN 1 is suspected, genetic mutations could not be found in all the exons of the MEN 1 gene that has been translated. To our knowledge, this is the second report of a multicentric type 3 gastric NET. More aggressive characteristics, including lymphovascular invasion, were observed in our case.

Type 3 gastric NETs exhibit a more aggressive course than the type 1 and 2 gastric NETs. Most type 3 gastric NETs showed lymphovascular and deep wall invasion at the time of diagnosis. ${ }^{20}$ In other cases, $60 \%$ to $75 \%$ present with metastatic disease, and tumor-related death occurs 28 months after diagnosis in $25 \%$ to $30 \%$ of cases. ${ }^{4,20}$ Thus, the therapeutic approach to type 3 gastric NETs should be managed surgically. ${ }^{9}$ Only small $(<10 \mathrm{~mm})$, well differentiated (G1) type 3 gastric NETs limited to the submucosal layer have been treated efficaciously by endoscopic mucosectomy, in a retrospective analysis. ${ }^{15}$

In summary, we described the case of a 50-year-old woman with multicentric type 3 gastric NET without hypergastrinemia, which was endoscopically similar to the type 1 and 2 lesions but with more aggressive features, such as lymphovascular invasion. It will be necessary to study various other cases in order to discover the pathogenesis, and develop therapeutic strategies. This case serves as a reminder to gastroenterologists who encounter NET cases in their clinical practice, that even if the NET is multiple, a diagnosis of type 3 NET should be considered so that a careful evaluation of atrophic status such as endoscopic findings, serologic biomarkers, and gastrin levels can be conducted.

\section{Conflicts of Interest}

The authors have no financial conflicts of interest.

\section{Acknowledgments}

I would like to express my very great appreciation to Dr. Lee, my supervisor, for his valuable and constructive suggestions during the planning and preparing of this case report.

\section{REFERENCES}

1. Modlin IM, Lye KD, Kidd M. A 5-decade analysis of 13,715 carcinoid tumors. Cancer 2003;97:934-959.

2. Lawrence B, Gustafsson BI, Chan A, Svejda B, Kidd M, Modlin IM. The epidemiology of gastroenteropancreatic neuroendocrine tumors. Endocrinol Metab Clin North Am 2011;40:1-18.

3. Modlin IM, Lye KD, Kidd M. A 50-year analysis of 562 gastric carcinoids: small tumor or larger problem? Am J Gastroenterol 2004;99:2332.

4. Rindi G, Luinetti O, Cornaggia M, Capella C, Solcia E. Three subtypes of gastric argyrophil carcinoid and the gastric neuroendocrine carcinoma: a clinicopathologic study. Gastroenterology 1993;104:994-1006.

5. Bordi C. Gastric carcinoids. Ital J Gastroenterol Hepatol 1999;31 Suppl 2:S94-S97.

6. Hassan MM, Phan A, Li D, Dagohoy CG, Leary C, Yao JC. Risk factors associated with neuroendocrine tumors: a U.S.-based case-control study. Int J Cancer 2008;123:867-873.

7. Delle Fave G, Capurso G, Annibale B, Panzuto F. Gastric neuroendocrine tumors. Neuroendocrinology 2004;80 Suppl 1:16-19.

8. Fléjou JF. WHO Classification of digestive tumors: the fourth edition. Ann Pathol 2011;31:S27-S31.

9. Delle Fave G, Kwekkeboom DJ, Van Cutsem E, et al. ENETS Consensus Guidelines for the management of patients with gastroduodenal neoplasms. Neuroendocrinology 2012;95:74-87.

10. Bordi C, D’Adda T, Azzoni C, Pilato FP, Caruana P. Hypergastrinemia and gastric enterochromaffin-like cells. Am J Surg Pathol 1995;19 Suppl 1:S8-S19.

11. Thomas D, Tsolakis AV, Grozinsky-Glasberg S, et al. Long-term follow-up of a large series of patients with type 1 gastric carcinoid tumors: data from a multicenter study. Eur J Endocrinol 2013;168:185-193.

12. Merola E, Sbrozzi-Vanni A, Panzuto F, et al. Type I gastric carcinoids: a prospective study on endoscopic management and recurrence rate. Neuroendocrinology 2012;95:207-213.

13. Spinelli P, Cerrai FG, Casella G, Pizzetti P. Endoscopic treatment of gastric carcinoids. Minerva Chir 1994;49:271-273.

14. Basuroy R, Srirajaskanthan R, Prachalias A, Quaglia A, Ramage JK. Review article: the investigation and management of gastric neuroendocrine tumours. Aliment Pharmacol Ther 2014;39:1071-1084.

15. Kwon YH, Jeon SW, Kim GH, et al. Long-term follow up of endoscopic resection for type 3 gastric NET. World J Gastroenterol 2013;19:87038708. 
16. Bordi C, Falchetti A, Azzoni C, et al. Aggressive forms of gastric neuroendocrine tumors in multiple endocrine neoplasia type I. Am J Surg Pathol 1997;21:1075-1082.

17. Hosoya Y, Fujii T, Nagai H, Shibusawa H, Tsukahara M, Kanazawa K. A case of multiple gastric carcinoids associated with multiple endocrine neoplasia type 1 without hypergastrinemia. Gastrointest Endosc 1999;50:692-695.

18. Katsinelos P, Chatzimavroudis G, Kotakidou R, Zavos C, Paroutoglou G,
Kountouras J. Diffuse gastric carcinoid polyposis in multiple endocrine neoplasia 1 syndrome without hypergastrinemia. Gastrointest Endosc 2007;66:411-412.

19. Moriyama T, Matsumoto T, Hizawa K, et al. A case of multicentric gastric carcinoids without hypergastrinemia. Endoscopy 2003;35:86-88.

20. Rindi G, Bordi C, Rappel S, La Rosa S, Stolte M, Solcia E. Gastric carcinoids and neuroendocrine carcinomas: pathogenesis, pathology, and behavior. World J Surg 1996;20:168-172. 